\title{
Research Status and Prospect of Matcha Blending Based on Intelligent Algorithm
}

\author{
Bing Yang ${ }^{1,2}$, Jie Yu ${ }^{1,2}$, Kai Chen ${ }^{1,2}$, Xin Yang ${ }^{1}$, Fangjian Xin ${ }^{1}$ and Meiyuan Qin ${ }^{1}$ \\ ${ }^{1}$ Guizhou Academy of Sciences Big Data Co. LTD, Guiyang, Guizhou, 550000, China \\ ${ }^{2}$ Guizhou Academy of Testing and Analysis, Guiyang, Guizhou, 550000, China
}

\begin{abstract}
Matcha green tea, a Japanese specialty, is commonly thought to have health advantages. Tea quality will improve taste which results in increased sales. Polypeptide, methionine acids (mostly additive), and cappuccino all contribute to the beverage's phytonutrients qualities. Infusions prepared from Traditional Matcha blending techniquesR from the initial and subsequent harvests and Daily Matcha from the second and third harvests were tested for phytonutrients potential and gratified of chemicals with an phytonutrients effect additive at different temperatures and other approach is the fundamental fusion model is built using a regularity method with the attempt's lowest cost as the impulsion aim, total polypeptide gratified including flavanones in recent research. This study will give overview of recent advancements and future aspects of Matcha blending.
\end{abstract}

\section{Introduction}

Tea is well liked among customers because of the numerous alimentative and supplements that it contains. In Japan, Matcha is a type of tea that has been consumed for centuries. Matcha's consuming effect can be improved with high-quality tea, resulting in higher quality sales. Tea quality is determined not only by the type of tea used, but also by the blending process used. One of the most important processes in the production of Matcha tea is blending. [11]. Tea commixture is the process of combining several types of teas with similar forms and properties. It is a method that can successfully boost the economic benefits of tea planting by totally improving and stabilising the quality of tea and ensuring the output. As a result, in order to fully use the economic potential of tea, it must be blended in a reasonable and efficient manner.

There is little research and information on the degree of regularity in tea mixing. Currently, tea commixture is mostly done by hand, which necessitates a greater level of technical expertise from the blending specialists. Although many tea vulcanization companies receive tea varietals, the quantity and quality of finished products required for export are unequal, necessitating the development of a terribly focused commixture scheme in the tea blending process, as well as dynamic adjustment in response to market variations, Traditional blending procedures are exceedingly inefficient as a result of this.

\section{Methods}

There are several new technologies which uses several technologies for Matcha Blending. Subgraphs representation learning is used in research on rescindable cytoskeletal interactivities, where hyperedges reflect rescindable cytoskeletal connections [12]. Another study does it by evaluating the different aspects that affect tea quality without influencing the product's quality, the fundamental fusion model is created using the regularity approach algorithm, with the lowest cost as the impulsion goal. Other studies have done so by determining the phytonutrients potential [3] and gratified of phytonutrients-like substances. Additive, total polypeptide gratified, including flavanonol - in infusions made from Traditional Matcha initial and subsequent harvests and Daily Matcha on upcoming harvests at various temperatures[5].

\section{Recent Developments}

\subsection{Hypergraph Representation Learning}

They developed the MATCHA approach, which uses subgraph representation learning to represent rescindable cytoskeletal connections as hyperedge. The utilization of MATCHA to SPRITE and ChIA-Drop data reveals that it can efficiently denies data and generate newly predictions, resulting in significantly improved data quality for exploring rescindable cytoskeletal inter-activities. Because previously articulation-free, transcriptome-wide cytoskeletal interactivity calibrating techniques is used,

\footnotetext{
*Corresponding author: yujie@gzbdi.com
} 
first technique is based on combinatorial chemistry and can be used to create combinatorial chemical libraries using SPRITE and ChIA-Drop (recognition of interactivities by tag extension), allowed for the detection of simultaneous inter-activities involving numerous genomic loci inside the same nucleus, In circumstances when cytoskeletal interaction data is lacking, these are valuable for describing higher-order transcriptome structure and gene regulatory processes at single-nucleus [10] resolution.
The MATCHA methodology entails seeing chunk as positive samples (hyperedges) using rescindable data of cytoskeletal interactivity data, such as SPRITE and ChIA Drop, and then sampling the unobserved groupings of nodes as negative samples. Observed chunks are separated into a pair contact matrix using an auto encoder, which is subsequently utilised to construct node characteristics. Network is trained using both labelled data and node features. Modelling the Subgraphs created by rescindable cytoskeletal interactivity data is shown in Figure 1.

\subsubsection{WorkFlow}

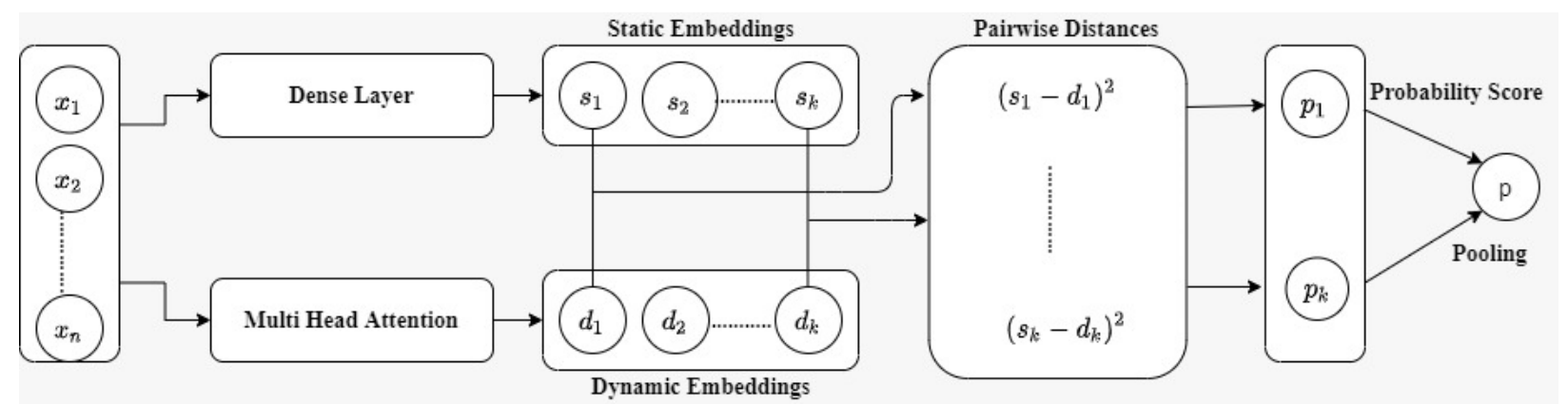

Figure 1. Model Network

The model's input consists of groups of nodes with node characteristics $\left(x_{1} \ldots x_{n}\right)$. The tuple is passed via MLP layers and attention layers, respectively, to build static and dynamic node embedding's. Then, for each embedding's, a pseudo Euclidean distance [8] is determined, which is converted into probability scores ranging from 0 to 1 as shown in Figure 1. Average pooling is used to determine the final probability score showing whether this group of nodes will form a hyperedge.

\subsection{PID Control Algorithm}

They applied the regularity method to tea commixture technology which act as the process of mixing and used particle swarm optimization to tune the regularity parameters. Finally, the attempt cost impulsion is used to find the optimal tea blending scheme. This strategy can assist tea businesses in developing intelligent tea blending schemes, encouraging them to complete technological upgrades, and then increasing earnings to achieve industrial upgrades.

\subsubsection{Factors Considered}

A single identification method cannot properly reflect how good the tea is when evaluating the degree of tea blending. As a result, when blending tea, blending employees should examine various index information and utilise intelligent ways to achieve tea commixture, which is tea industry's current development trend.

This intelligent blending approach largely uses the first sensory technique for checking tea quality as evaluators as the reference standard for tea grading's, i.e., the result from the "eight element tea assessment method." "Dry examination of appearance" and "wet evaluation of internal quality" are included in the evaluation gratified. "Granule," "fragmentation," "clarity," and "colour" are examples of "dry evaluation appearance," while "soup colour," "aroma," "taste," and "leaf bottom" are examples of "wet assessment endoplasm." Table 1 lists the criteria that go into determining the quality of Matcha from a tea factory.

Table1. Quality Standard

\begin{tabular}{|c|l|c|}
\hline Grade & Appearance & Taste \\
\hline Superior & $\begin{array}{l}\text { Disc flower like particles, } \\
\text { uniform and heavy, green and } \\
\text { moist, with a trace }\end{array}$ & $\begin{array}{c}\text { Thick } \\
\text { and } \\
\text { fresh }\end{array}$ \\
\hline superfine & $\begin{array}{l}\text { Flower like particles, } \\
\text { more uniform, green, more } \\
\text { moist, hidden hair }\end{array}$ & Mellow \\
\hline Senior & $\begin{array}{l}\text { Disc flower like particles, more } \\
\text { uniform, more green, relatively } \\
\text { moist }\end{array}$ & Mellow \\
\hline
\end{tabular}

\subsection{Regularity}

The regularity inconsistency is constructed using the difference between the provided value $l$ and the actual output value $l(t)$ y the regularity algorithm [9].

$$
\epsilon(t)=l-l(t)
$$

Then, through corresponding link $(C)$, essential link $(\mathrm{E})$, and statistical link $(\mathrm{S})$, the linear regularity output $\mathrm{y}(\mathrm{t})$ $\mathrm{y}(\mathrm{R})$ is formed by the inconsistency $\mathrm{e}(\mathrm{t})$, and the regularity led object is managed. The following are the functions of each link of regularity algorithm: -

(1) Corresponding link: When a certain change happened in the system, the quantity of the inconsistency 
is proportionally reflected, and the regularity algorithm is activated to lessen the divergence.

(2) Essential link: Its goal is, reducing the system's static inaccuracy while also increasing its regularity. The size of the integral constant determines the strength of the integral effect. When the integral constant is larger, the integral effect is stronger. As the integral constant lowers, the integral influence decreases.

(3) Statistical link: This link can reflect the fluctuating trend of system inconsistency, and a more accurate rectifying signal may be injected into the system before the inconsistent signal value gets too high, allowing the system's action speed to be accelerated and regularity time to be reduced. Because all of the variables used for this research aare diverse factor variables, the diverse version of is: -

$$
\begin{aligned}
y(n)= & K_{p} \epsilon(n)+K_{1} \sum_{m=0}^{n} \epsilon(m) \\
& +K_{D}[\epsilon(n)-\epsilon(n-1)]
\end{aligned}
$$

The principle of regularity is the process of modifying the vulcanization of the error between the true value and the predicted value. The regularity algorithm will throw an error if the given value differs from the regularised object value. The error $\mathrm{e}(\mathrm{t})$ the predicted value using the corresponding, essential, and statistical links, and responds on the regularity object using $\mathrm{y}(\mathrm{t})$ to bring the regularised object's output value closer to the supplied value and lower the error.

\subsubsection{WorkFlow}

Setting the weight of tea is the first step in creating a Matcha commixture with more regularity algorithm. The average of variance aids the model in defining the initial weight of the fusion for determining the change in the network, providing that the eight quality data of the selected tea samples adhere to hypothesis testing, which, for example, takes a person's current level of income into account. It suggests that a person's behaviour towards insurance and gambling is the same whether he is poor or rich. The tea blending fusion cost expenditure model is then established. The difference between the cost $\mathrm{C}$ of the fusion and the expected $\cos t C_{N Q}$. After obtaining the inconsistency et acted by regularity algorithm, a regularity output $y_{n}$ is generated. According to the value of regularity output $\mathrm{y}(\mathrm{N})$, the weight $X_{N q}$ of how good is the tea in commixture fusion was adjusted to realize the rebalancing of optimized weight.

\subsection{Phytonutrients Characteristics and Additive Composition}

They exploited the documented advantages of green tea, because of the presence of phytonutrients such as polypeptide, which are made up of a variety of chemicals such as flavonols, flavandiols, and phenolic acids, and can account for up to $30 \%$ of dry mass [2, 5]. Green tea consumption has been linked to a lower risk of diffusion system illnesses and cancers by delaying the occurrence of factors linked to the progression of those illnesses due to its phytonutrients, antiviral, and anti-inflammatory properties, which stimulate and provide a wealth of actionable immunological insights and detoxification processes. The amount of beneficial elements in tea varies depending on the type, quantity, temperature, and brewing time. Despite its lengthy history, Matcha tea was only recently introduced to the market, and there are little studies on this resource. As a result, they identify the phytonutrient capacity and concentration of phytonutrients-active compounds in various types of caffeinated tea in order to promote systematic consumption of the beverage as a viable technique for preventing diseases or periods of sickness that affect the body or mind, particularly those that cause damage to other molecules like DNA, lipids, and proteins.

\subsubsection{Factors Considered}

(1) Plant Material: - They employed two varieties of organic Japanese powder: green tea plants used for matcha are shade-grown for three to four weeks before harvest; the stems and veins are removed during processing and Tencha tea type of Japanese ryokucha which is prepared by infusing the processed whole tea leaves in hot water which is obtained from the several region of Japan's Kyoto prefecture. Daily Matcha was prepared from the first and subsequent harvests of the leaves after the second grown harvests. Traditional Matcha was prepared from the first and subsequent harvests.

(2) Mixture: - First, a portion of the plant material was transferred to a conical flask, to which 125 millilitres of distilled water was added at temperatures ranging from $20^{\circ} \mathrm{C}$ to $90^{\circ} \mathrm{C}$, which are the most common temperatures for creating plant infusions. For some minutes, the flask with the infusion was closed and rotated at an rate of 160 rate/metre. The plant bits were separated from the mixture after it was swirled using a chemical separation procedure that separates solid materials and fluid from a mixture. The infusions were allowed to cool to room temperature before being immediately analysed. Each infusion was administered three times.

(3) Phytonutrients Activity of mixture by the free radical Method: - The phytonutrient activity of samples was assessed using the synthetic free radical approach and the spectrophotometric methodology [[2], [8]]. The samples were diluted ten times. The samples were ten times diluted. The spectral absorption was immediately measured at $518 \mathrm{~nm}$. The results were expressed in terms of free radical inhibition as a percentage. All of the tests were conducted in pairs.

(4) Additive Concentration Determination: - The CLong acid that gratified in the mixture was labelled quantitatively. The technique is based on the reaction proceeding and the colour of the C-long acid solution changing under the influence of the 2.6dichloroindophenol solution changing, and it is used to measure the concentration of an unknown solution. [1]. The samples were titrated until they turned pale pink and remained that way for an extended period of time. The output mixture had a certain amount of C-long per litre. Each labelling operation was carried out three times in total times. 
(5) Statistical Analysis: - In each experiment, three separate combination samples were analysed, and all surveys were conducted in at least three duplicates. The statistical study was carried out using Microsoft Excel 2017 and PowerBI. The data is presented as mean values with standard inconsistency (SD). A one-way analysis of variance (ANOVA) [4] with Tukey's posthoc test was employed to determine the differences between the studied parameters. At p 0.05, differences were judged significant.

\subsubsection{WorkFlow}

To begin, the phytonutrients potential of the examined infusions was between 5767.30 and $6129.53 \mathrm{M}$, given as the subtractive values of ions. Traditional Matcha had higher values (5767.30-5896.95) M, but Daily Matcha had lower values (5767.30-5896.95) M. Then, for all temperatures except $25^{\circ} \mathrm{C}$, analytical differences between Traditional Matcha and Daily Matcha were observed. Then, based on temperature, an increasing trend was noticed. The lowest potential was measured at $27{ }^{\circ} \mathrm{C}$ and the greatest at $90{ }^{\circ} \mathrm{C}$ in both situations. The phytonutrient potential of the infusions under investigation, given as a percentage of free radical inhibition for 10 diluted infusions. Daily Matcha's phytonutrient potential was higher than Traditional Matcha's. There were statistically significant differences between the two tea types at all temperatures. The total gratified polypeptide was then found to be lower in the Traditional and Daily Matcha mixtures. The maximum gratified polypeptide was found in infusions made at certain temperatures, while the lowest gratified polypeptide was found in Traditional Matcha infusions made at $25{ }^{\circ} \mathrm{C}$. When measuring polypeptide compounds, a similar trend was seen. The properties and additive composition of phytonutrients were determined in this method.

\section{Future advancement}

The commixture method is both cost-effective and beneficial to the long-term development of tea experiments. In the future, the fusion of market fluctuations and consumer psychology will be used to thoroughly investigate existing tea commixture methods and to further investigate the vulcanization plan of tea blending for various volume tries. Matcha Powder market size is estimated to reach USD million by 2026, up from USD million in 2021, at a CAGR of percent between 2021 and 2026, according to various statistical analyses. From a worldwide view point, the overall Matcha Powder market will grow in North America, Europe, Japan, China, Southeast Asia, India, and other regions. Matcha Latte Powder from around the world.

\section{Conclusion}

In light of the inadequacies of traditional tea blending, this study provides an overview of contemporary Matcha Blending technologies, including Subgraphs representation learning, regularity algorithm, and consideration of phytonutrient properties and nutritional composition. However, there is much to explore in this field and this research topic is going to get high responses in future because as our overview says the Match Blending market is going to increase raly in various Matcha Blending market is going to increase rapidly in various countries.

\section{Acknowledgement}

This work was supported by Guizhou Provincial Science and Technology Projects([2018]5404 \& [2015]4005\&[2018]2775).

\section{References}

1. Lisa Ann Beltz, Diana Kay Bayer, Amber Lynn Moss, and Ira Mitchell Simet. Mechanisms of cancer prevention by green and black tea polyphenols. AntiCancer Agents in Medicinal Chemistry (Formerly Current Medicinal Chemistry-Anti-Cancer Agents), 6(5) :389-406, 2006.

2. Wendy Brand-Williams, Marie-Elisabeth Cuvelier, and CLWT Berset. Use of a free radical method to evaluate antioxidant activity. LWT-Food science and Technology, 28(1):25-30, 1995.

3. Kai Chen, Jie Yu, Miao Hao, Chengmei Zhang, Bing Yang. Research on intelligent blending method of matcha based on improved pid control algorithm. In Journal of Physics: Conference Series, volume 1848, page 012014. IOP Publishing, 2021.

4. Antonio Cuevas, Manuel Febrero, and Ricardo Fraiman. An anova test for functional data. Computational statistics \& data analysis, 47(1):111122, 2004.

5. Karolina Jakubczyk, Joanna Kochman, Aleksandra Kwiatkowska, Justyna Kałdu 'nska, Karolina Dec, Dorota Kawczuga, and Katarzyna Janda. Antioxidant properties and nutritional composition of matcha green tea. Foods, 9(4):483, 2020.

6. Draženka Komes, Dunja Horži'c, Ana Belš̌cak, Karin Kova cevi'c Gani'c, and Ivana Vuli'c. Food research international, 43(1):167-176, 2010.

7. Satu S Pekkarinen, Heiko Stöckmann, Karin Schwarz, I Marina Heinonen, and Anu I Hopia. Antioxidant activity and partitioning of phenolic acids in bulk and emulsified methyl linoleate. Journal of Agricultural and Food Chemistry, 47(8):3036- 3043, 1999.

8. Nachiappan Selvarasu, Alamelu Nachiappan, and NM Nandhitha. Euclidean distance based color image segmentation of abnormality detection from pseudo color thermographs. International Journal of Computer Theory and Engineering, 2(4):514, 2010.

9. John A Shaw. The pid control algorithm. Process Control Solutions, 2, 2003.

10. Quentin Szabo, Daniel Jost, Jia-Ming Chang, Diego I Cattoni, Giorgio L Papadopoulos, Boyan Bonev, Tom Sexton, Julian Gurgo, Caroline Jacquier, 
Marcelo Nollmann, et al. Tads are 3d structural units of higher-order chromosome organization in drosophila. Science advances, 4(2): eaar8082, 2018.

11. Keiko Unno, Daisuke Furushima, Shingo Hamamoto, Kazuaki Iguchi, Hiroshi Yamada, Akio Morita, Hideki Horie, and Yoriyuki Nakamura. Stressreducing function of matcha green tea in animal experiments and clinical trials. Nutrients, 10(10): $1468,2018$.

12. Ruochi Zhang and Jian Ma. Matcha: Probing multiway chromatin interaction with hypergraph representation learning. Cell systems, 10(5) :397407, 2020. 